\title{
Cholesteryl Ester Hydroperoxide Formation in Myoglobin-Catalyzed Low Density Lipoprotein Oxidation
} Concerted Antioxidant Activity of Caffeic and p-Coumaric Acids
with Ascorbate

\author{
Otília Vieira, ${ }^{*} \dagger$ João Laranjinha, ${ }^{*} \dagger$ Vitor Madeira $\dagger$ and Leonor Almeida*† \\ *Laboratory of Biochemistry, Faculty of Pharmacy; and †Center for Neurosciences, \\ University of Coimbra, 3000 Coimbra, Portugal
}

\begin{abstract}
Two diet-derived phenolic acids, caffeic and p-coumaric acids, interplayed with ascorbate in the protection of low density lipoproteins (LDL) from oxidation promoted by ferrylmyoglobin. Ferrylmyoglobin, a two-electron oxidation product from the reaction of metmyoglobin and $\mathrm{H}_{2} \mathrm{O}_{2}$, was able to oxidize LDL, degrading free cholesterol and cholesteryl esters. Upon exposure to ferrylmyoglobin, LDL became rapidly depleted of cholesteryl arachidonate and linoleate, which turn into the corresponding hydroperoxides. Cholesteryl oleate and cholesterol were, comparatively, more resistant to oxidation. Caffeic $(2 \mu \mathrm{M})$ and p-coumaric $(12 \mu \mathrm{M})$ acids efficiently delayed oxidations, as reflected by an increase in the lag times required for linoleate hydroperoxide and 7-ketocholesterol formation as well as for cholesteryl linoleate consumption. At the same concentration, ascorbate, a standard water-soluble antioxidant, was less efficient than the phenolic acids. Additionally, phenolic acids afforded a protection to LDL that, conversely to ascorbate, extends along the time, as inferred from the high levels of cholesteryl linoleate and cholesteryl arachidonate left after $22 \mathrm{hr}$ of oxidation challenging. Significantly, the coincubation of LDL with ascorbate and each of the phenolic acids resulted in a synergistic protection from oxidation. This was inferred from the lag phases of cholesteryl linoleate hydroperoxide (the major peroxide found in LDL) formation in the presence of mixtures of ascorbate with phenolic acids longer than the sum of individual lag phases of ascorbate and the phenolic acids. A similar description could be drawn for the accumulation of a late product of oxidation, 7-ketocholesterol. It is concluded that ferrylmyoglobin induces a typical pattern of LDL lipid peroxidation, the oxidation rate of cholesteryl esters being a function of unsaturation; furthermore, there is a synergistic antioxidant activity of diet-derived phenolic acids with ascorbate in the protection of LDL from oxidation, a finding of putative physiological relevance. BIOCHEM PHARMACOL 55;3:333-340, 1998. (C) 1998 Elsevier Science Inc.
\end{abstract}

KEY WORDS. LDL; ferrylmyoglobin; lipid peroxidation; antioxidant synergism; ascorbate; caffeic acid; p-coumaric acid

There is increasing evidence that oxidation of plasmatic low density lipoproteins (LDL) $\S$ is a critical factor in promoting atherosclerosis [1]. Cholesterol and cholesteryl esters are the main lipid components of human LDL [2] and, apparently, these compounds are oxidized more rapidly to hydroperoxides than the polyunsaturated phospholipids in the outermonolayer [3, 4]. Thereafter, hydroperoxides can decompose to reactive aldehydes that in turn may modify amino acid residues of the apoB. The LDL particles

\footnotetext{
* Corresponding author: Dr. João Laranjinha, Laboratory of Biochemistry, Faculty of Pharmacy, University of Coimbra, Couraça dos Apóstolos. 51, r/c, 3000 Coimbra, Portugal. FAX +351-39-27940; Tel. +351-39-22788; E-mail: laranjin@cygnus.ci.uc.pt

§Abbreviations: LDL, low density lipoproteins; CL-OOH, cholesteryl linoleate hydroperoxide; $\mathrm{CH}$, free cholesterol; $\mathrm{CA}$, cholesteryl arachidonate; CL, cholesteryl linoleate; $\mathrm{CO}$, cholesteryl oleate; 7-KC, 7-ketocholesterol; AAPH, 2,2' -azo-bis-(2-amidinopropane)hydrochloride; BHT, butylated hydroxytoluene; DTPA, diethylenetriaminepentacetic acid.

Received 30 May 1997; accepted 30 July 1997.
}

containing oxidatively modified apoprotein are recognized, in the arterial wall, by the scavenger receptors in macrophages leading to uncontroled intracellular lipid accumulation and transformation of the cells into lipid-laden foam cells, which characterize the early lesions of atherosclerosis [5]. On the other hand, oxygenated derivatives of free cholesterol (oxysterols) possess a wide variety of biological properties, including cytotoxicity [6]. Thus, the study of LDL lipid peroxidation and its inhibition by antioxidants is of considerable interest in the molecular mechanism of atherosclerosis and in the design of active drugs to prevent the disease.

Ferrylmyoglobin, a two-electron oxidation product from the reaction of metmyoglobin with $\mathrm{H}_{2} \mathrm{O}_{2}$, initiates lipid peroxidation in $\mathrm{LDL}$, a process with putative significance in vivo [7]. This strong oxidant derivative of myoglobin contains two oxidizing centers, a relatively stable hypervalent iron $\left(\mathrm{Fe}^{\mathrm{IV}}=\mathrm{O}\right)$, and a transient radical in the globin 
moiety [for review see 8], but detailed mechanisms and the dynamics of the oxidation of LDL induced by ferrylmyoglobin are still incipient. This hypervalent derivative of myoglobin may be of physiological relevance in the ensuing damage to LDL during the development of atherosclerosis. On the other hand, a considerable effort is currently being made to evaluate natural compounds that may act as protective agents against oxidation of biological systems. Recently, we reported that lipid peroxidation in LDL induced by ferrylmyoglobin is prevented by dietary phenolic acids, namely caffeic and p-coumaric acids [9]. The underlying mechanisms encompass the reduction of ferrylmyoglobin to harmless metmyoglobin [10]. These compounds are ubiquitously present in fruits and vegetables and may be found in human plasma as a function of diet concentration [11]. Moreover, there is in vitro evidence for the scavenging activity of phenolic acids against free radicals and chain-breaking antioxidant activity in LDL particles [12]. However, the interplay of natural and "physiological" antioxidants has not been addressed. Only recently, the synergistic interaction of caffeic acid and $\alpha$ tocopherol, in the protection of LDL from oxidation, explained in terms of the regeneration of $\alpha$-tocopherol from its radical, has been reported [13].

This work describes the time-courses of cholesterol, cholesteryl linoleate, cholesteryl oleate and cholesteryl arachidonate oxidations as well as the formation of cholesteryl linoleate hydroperoxide and 7-ketocholesterol in LDL challenged with ferrylmyoglobin, in an attempt to understand the dynamics of ferrylmyoglobin-induced oxidation. We also describe the concerted activity of selected natural antioxidants, caffeic and p-coumaric acids, with ascorbate on the rate of cholesteryl linoleate hydroperoxide and 7-ketocholesterol formation in LDL peroxidation induced by ferrylmyoglobin. A synergistic antioxidant effect was shown to arise from the interplay of ascorbate with the phenolic acids. The concerted electron transfer to ferrylmyoglobin by each of the antioxidants, reducing it to harmless metmyoglobin, is an eventual molecular mechanism underlying this effect, but reduction of caffeic and $p$-coumaric acid radicals by ascorbate is suggested to occur.

\section{MATERIALS AND METHODS Reagents}

Horse heart metmyoglobin, the phenolic acids, cholesterol, 7-ketocholesterol and the cholesteryl esters were obtained from Sigma Chemical Co. Hydrogen peroxide was purchased from Merck as a 30\% solution. Ascorbate was obtained from Fluka Chemika. Tetrasulphonated chloraluminium phtalocyanine was a gift from Ciba-Geiger. Before use, metmyoglobin was purified by dialysis against phosphate buffer ( $20 \mathrm{mM}$ phosphate, $110 \mathrm{mM} \mathrm{NaCl}$ ), pH 7.4 (referred to as PBS) containing DTPA and Chelex. Stock metmyoglobin and hydrogen peroxide solutions were standardized using $\epsilon_{632 \mathrm{~nm}}=2.1 \mathrm{mM}^{-1} . \mathrm{cm}^{-1}$ and $\epsilon_{240 \mathrm{~nm}}=43.6$ $\mathrm{M}^{-1} . \mathrm{cm}^{-1}$, respectively. All solutions were prepared in water purified in a Milli-Q apparatus. Ascorbate and phenolic acids were dissolved in water.

\section{LDL Isolation}

LDL particles were isolated from fresh human blood of healthy normolipidemic volunteers by a rapid isolation method [14] using a Beckman L80 ultracentrifuge (Beckman Inst. Inc.) equipped with a Beckman 70.1 Ti fixed angle rotor. The isolation of LDL, achieved by a discontinuous gradient density step (180 min), was followed by a dialysis (against PBS) and a simultaneous concentration step (45 min) by ultrafiltration through a collodium bag, under nitrogen. Then, the LDL samples were sterile-filtered through a $0.22-\mu \mathrm{m}$ filter (Millipore), stored in the dark at $4^{\circ}$ and used for the experiments within one week. The protein content of LDL samples was measured according to Lowry [15] using bovine serum albumin (BSA) as standard, and the purity of LDL preparation was checked by SDSpolyacrylamide gradient (3-20\%) gel electrophoresis.

\section{Oxidation Conditions}

LDL samples $(200 \mu \mathrm{g} / \mathrm{mL})$ in PBS, $\mathrm{pH} 7.4$, were incubated at $37^{\circ}$ under gentle stirring, and the oxidation reaction was initiated by addition of $6 \mu \mathrm{M}$ metmyoglobin $/ 9 \mu \mathrm{M} \mathrm{H}_{2} \mathrm{O}_{2}$ in the absence or presence of phenolic acids at the concentrations given in legends to figures. All the incubations were performed in presence of $100 \mu \mathrm{M}$ DTPA (stock solution in PBS) to quelate trace transition metals, preventing hydroperoxide decomposition from Fenton-type reactions. The oxidation reactions were stopped by addition of $20 \mu \mathrm{M}$ butylated hydroxytoluene (final concentration) from stock solution prepared in ethanol. LDL oxidation was followed by the consumption of cholesterol and cholesteryl esters and cholesteryl linoleate hydroperoxide (CL-OOH) formation.

\section{Analysis of Cholesterol, Cholesteryl Esters and Cholesteryl Linoleate Hydroperoxide}

Cholesterol, cholesteryl esters and cholesteryl linoleate hydroperoxide in LDL samples were determined by HPLC essentially as described by Kritharides et al. [16]. Briefly, 1 $\mathrm{mL}$ aliquots (200 $\mu \mathrm{g}$ of LDL protein) were withdrawn along the incubation time, chilled in ice, spiked with $20 \mu \mathrm{M}$ BHT, and lipids extracted with methanol and $n$-hexane. The hexane extracts were analyzed by HPLC on a Beckman-System Gold consisting of a 126 Binary Pump Model and a 166 Variable UV Detector controled by a computer. The column was a LiChrospher $100 \mathrm{RP}-18(5 \mu \mathrm{m})$ from Merck. Cholesterol and cholesteryl esters were eluted with a solvent mixture consisting of acetonitrile/isopropanol $(30 / 70, v / v)$, and cholesteryl linoleate hydroperoxide $(\mathrm{CL}-\mathrm{OOH})$ and cholest-5-en-3 $\beta$-ol-7-one (7-ketocholesterol, 7-KC) were eluted with acetonitrile/isopropanol/ water $(44 / 54 / 2, \mathrm{v} / \mathrm{v} / \mathrm{v})$ at a flow rate of $1 \mathrm{~mL} / \mathrm{min}$. Analysis 


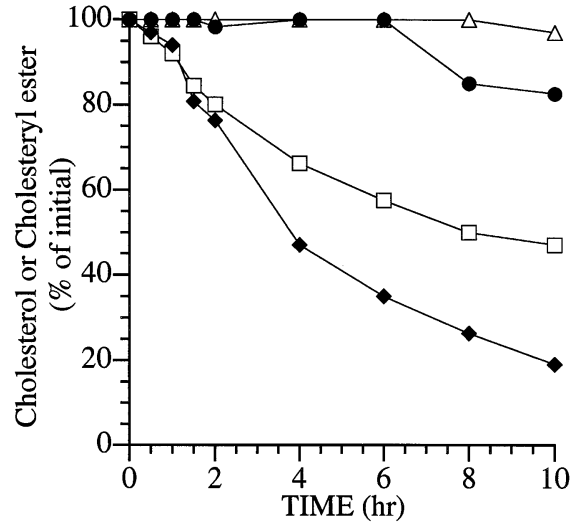

FIG. 1. Time-courses of cholesterol and cholesteryl ester degradation during oxidation of LDL by ferrylmyoglobin. LDL (200 $\mu \mathrm{g}$ protein $/ \mathrm{mL}$ ) were incubated with $6 \mu \mathrm{M}$ metmyoglobin $/ 9 \mu \mathrm{M}$ $\mathrm{H}_{2} \mathrm{O}_{2}$ in PBS containing DTPA $(100 \mu \mathrm{M})$ for $10 \mathrm{hr}$, at $37^{\circ}$. Free cholesterol $(\triangle)$, cholesteryl arachidonate $(\diamond)$, cholesteryl linoleate $(\square)$ and cholesteryl oleate $(\bullet)$ were analyzed by HPLC along the oxidation time, and the amounts are expressed relative to initial values before starting oxidations. Each point represents the mean of triplicate determinations of separate experiments performed with the same LDL preparation.

of these compounds was performed by UV detection at 210 or $234 \mathrm{~nm}$ for cholesterol and cholesteryl esters or cholesteryl linoleate hydroperoxide and 7-ketocholesterol, respectively.

CL-OOH standard was prepared photochemically by irradiating the solution of cholesteryl linoleate in the presence of tetrasulphonated chloraluminium phtalocyanine, as described by Thomas et al. [17] and quantified according to Saadani et al. [18], using the molar absorptivity of iodine measured at $365 \mathrm{~nm}\left(\epsilon=2.46 \pm 0.25 \times 10^{4}\right.$ $\left.\mathrm{M}^{-1} \cdot \mathrm{cm}^{-1}\right)$. This assay makes use of the oxidative capacity of lipid peroxides to convert iodide to iodine, which can be measured spectrophotometrically at $365 \mathrm{~nm}$.

\section{RESULTS}

Time-Courses of Free Cholesterol and Cholesteryl Ester Degradation and of Cholesteryl Linoleate Hydroperoxide Formation in LDL Challenged with Ferrylmyoglobin

Oxidation of LDL promoted by ferrylmyoglobin induced a rapid consumption of both cholesteryl arachidonate and linoleate, as compared with the consumption of cholesteryl oleate or free cholesterol (Fig. 1). Whereas cholesteryl arachidonate and cholesteryl linoleate decreased after the addition of ferrylmyoglobin (produced from metmyoglobin/ $\mathrm{H}_{2} \mathrm{O}_{2}$ mixture), cholesteryl oleate levels remained unchanged for $6 \mathrm{hr}$ and the degradation of free cholesterol was negligible during the time of the experiment. After $10 \mathrm{hr}$, cholesteryl arachidonate, linoleate and oleate in oxidized LDL were 19,47 and $82 \%$ of the initial values, respectively. Therefore, the consumption rate was a function of the degree of unsaturation of the ester acyl chain. These oxidation profiles, in terms of consumption of cholesterol and cholesteryl esters, promoted by ferrylmyoglobin in LDL

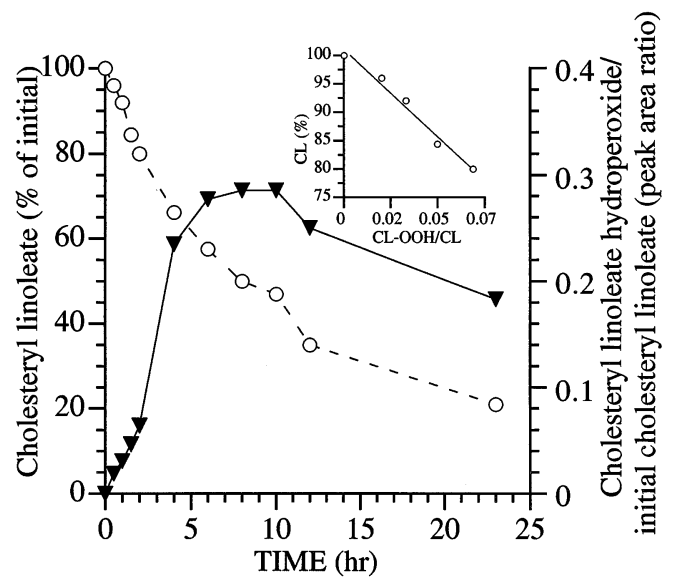

FIG. 2. Cholesteryl linoleate hydroperoxide formation (solid line) and contents of cholesteryl linoleate (dashed line) in LDL oxidized by ferrylmyoglobin. LDL aliquots $(1 \mathrm{~mL})$ were withdrawn from the reaction mixture for lipid extraction and measurement of cholesteryl linoleate and cholesteryl linoleate hydroperoxide by HPLC. Cholesteryl linoleate (CL) is expressed relative to the initial value before starting oxidation, and cholesteryl linoleate hydroperoxide is expressed as the ratio of formed CL-OOH and the initial CL, as determined by the ratio of the respective HPLC peak areas. The inset shows the linear relationship of CL vs. CL-OOH up to $2 \mathrm{hr}$ of reaction $(r=$ 0.98). Values represent the means of triplicate independent experiments.

were qualitatively similar to those promoted by AAPH (results not shown), a reference oxidant that generates peroxyl radicals in the aqueous phase at a constant rate [19].

Cholesteryl linoleate hydroperoxide (CL-OOH), not detected in the original LDL fraction, increased in parallel with the loss of cholesteryl linoleate (CL), up to $6 \mathrm{hr}$ of oxidation challenge (Fig. 2). During the initial period (2 hr), CL was exclusively converted to the respective hydroperoxide, as indicated by the high correlation coefficient $(r=0.98)$ of linear regression analysis of formed cholesteryl linoleate hydroperoxide vs. cholesteryl linoleate remaining in LDL particles (Fig. 2 inset). After a period of $8 \mathrm{hr}$, the relationship deviated from linearity, probably due to $\mathrm{CL}-\mathrm{OOH}$ decomposition. When the content of CL$\mathrm{OOH}$ reached its maximum value, a large amount of $\mathrm{CL}$ still remained (ca. $50 \%$ of the initial amount).

Effects of Phenolic Acids and Ascorbate on the TimeCourses of Cholesteryl Ester Consumption and Cholesteryl Linoleate Hydroperoxide Formation

Under similar experimental conditions, the presence of caffeic or p-coumaric acids, before addition of ferrylmyoglobin to LDL solution, led to the inhibition of $\mathrm{CL}$ consumption (Fig. 3A) and CL-OOH formation (Fig. 3B) as a function of concentration. Caffeic acid, the most efficient antioxidant, induced at $2 \mu \mathrm{M}$ a lag phase of $c a .6$ or $4 \mathrm{hr}$ in the consumption of cholesteryl linoleate (Fig. $3 \mathrm{~A}$ ) or in the hydroperoxide formation (Fig. 3B), respectively, an effect only observed at $12 \mu \mathrm{M} p$-coumaric acid. 

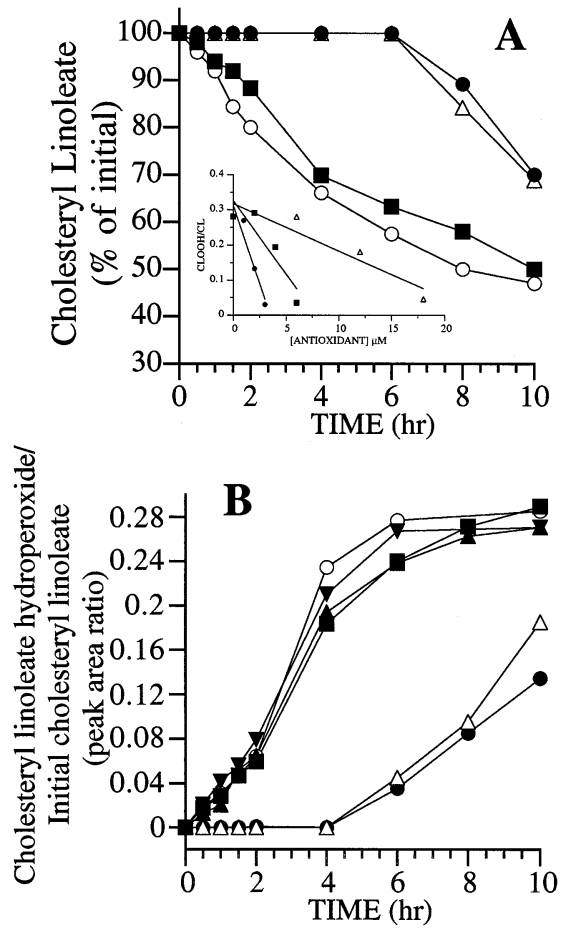

FIG. 3. Effects of caffeic acid, p-coumaric acid and ascorbate on the rates of cholesteryl linoleate contents (A) or cholesteryl linoleate hydroperoxide formation (B) in LDL oxidized by ferrylmyoglobin. Aliquots were withdrawn from the reaction mixture, processed, and analyzed by HPLC. Symbols: $(\bigcirc)$ control (no antioxidant addition), $(\circlearrowleft)$ caffeic acid $(2 \mu \mathrm{M}),(\triangle)$ p-coumaric acid $(12 \mu \mathrm{M}),(\boldsymbol{\square})$ ascorbate $(2 \mu \mathrm{M}),(\boldsymbol{\Delta})$ caffeic acid $(1 \mu \mathrm{M})$ and $(\nabla) p$-coumaric acid $(6 \mu \mathrm{M})$. The antioxidants were added $3 \mathrm{~min}$ before starting the oxidation reaction with $\mathrm{H}_{2} \mathrm{O}_{2}$. Each point represents the mean of duplicate determinations in independent experiments. Cholesteryl linoleate and the respective hydroperoxide are expressed as indicated in the legend to Fig. 2. A dose-response curve for the three antioxidants is included as inset to Fig. 3A. Measurements were made after 10 hr incubation. A linear correlation coeficient $>0.90$ was achieved for all the compounds. Each point represents the mean of two independent measurements within an experimental error of $5 \%$.

Moreover, after this lag phase, along the incubation time, a strong depression of the hydroperoxide formation rate was observed (Fig. 3B). The incubation of LDL with metmyoglobin (without $\mathrm{H}_{2} \mathrm{O}_{2}$ ) in the presence of $p$-coumaric or caffeic resulted in the complete prevention of CL loss.

From Fig. 3 it is clear that, for identical concentrations (2 $\mu \mathrm{M})$, caffeic acid exhibited a significantly higher protective effect against ferrylmyoglobin-mediated LDL oxidation over ascorbate, the major water-soluble antioxidant in plasma [20]. Indeed, $2 \mu \mathrm{M}$ ascorbate only slightly inhibited $\mathrm{CL}-\mathrm{OOH}$ formation, comparable to the effects of $1 \mu \mathrm{M}$ caffeic or $6 \mu \mathrm{M}$ p-coumaric acid (Fig. 3B). Conversely to 2 $\mu \mathrm{M}$ caffeic acid, $2 \mu \mathrm{M}$ ascorbate did not effectively inhibit ferrylmyoglobin-mediated LDL oxidation; after $10 \mathrm{hr}$ incubation, the cholesteryl linoleate loss in LDL particles amounted to 50 and $53 \%$ of total in the presence or absence of $2 \mu \mathrm{M}$ ascorbate, respectively (Fig. 3A). To better

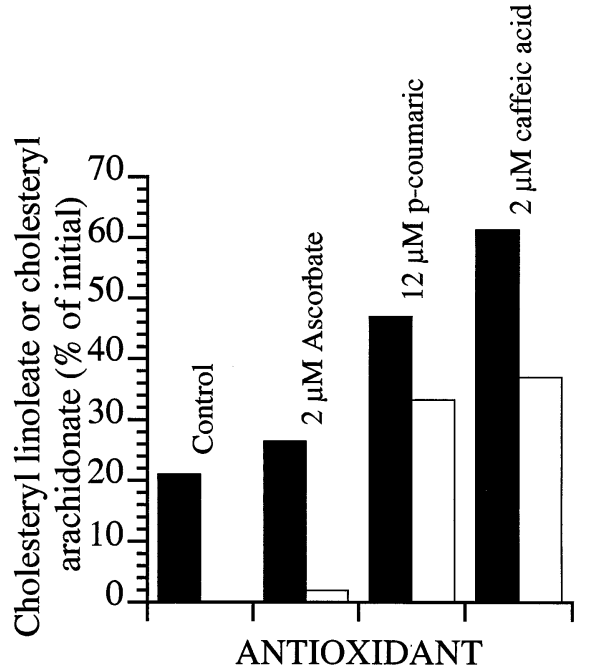

FIG. 4. Effect of caffeic acid, p-coumaric acid and ascorbate on the contents of cholesteryl arachidonate (white bars) or linoleate (black bars) remaining in LDL at $22 \mathrm{hr}$ incubation in the presence of ferrylmyoglobin. Results are expressed relative to the initial contents of esters before starting oxidation. In the control assay (absence of phenolic acids), cholesteryl arachidonate is below the detection limit. When present, phenolic acids were added, at the indicated concentrations, $3 \mathrm{~min}$ before starting the oxidation reaction. Error bars are not given since the values are taken from a single representative set of experiments with the same LDL aliquot and stock solutions of phenolic acids, ascorbate, metmyoglobin and $\mathrm{H}_{2} \mathrm{O}_{2}$. Experimental uncertainties were negligible.

evaluate the independent antioxidant activities of ascorbate, caffeic and p-coumaric acids, a dose-response curve based on the accumulation of $\mathrm{CL}-\mathrm{OOH}$ is included as an inset to Fig. 3A. The estimated $\mathrm{IC}_{50}$ values of caffeic acid, ascorbate and p-coumaric acid, within an experimental error of $5 \%$, were $1.9,5.2$ and $14.4 \mu \mathrm{M}$, respectively.

After $22 \mathrm{hr}$ oxidation (Fig. 4) challenged by $6 \mu \mathrm{M}$ metmyoglobin/9 $\mu \mathrm{M} \mathrm{H}_{2} \mathrm{O}_{2}$ (corresponding to an extensive LDL oxidation), approximately only $20 \%$ of CL was left in LDL and CA was not detected; ascorbate did not effectively prevent these losses. In fact, 73.5 and $98 \%$ of cholesteryl linoleate and cholesteryl arachidonate, respectively, were lost despite the presence of $2 \mu \mathrm{M}$ ascorbate. In contrast, only 39 and $63 \%$ of the same esters were lost when $2 \mu \mathrm{M}$ caffeic acid was present. In general, the differences between the protective effects of $2 \mu \mathrm{M}$ caffeic acid and $12 \mu \mathrm{M}$ $p$-coumaric acid were minor and not significant. The results summarized in Fig. 4 point out that the protection afforded by these phenolic acids extended along the time, in contrast to the effect of ascorbate that was negligible at later times.

Moreover, caffeic and p-coumaric acids, at the indicated concentrations, fully protected the degradation of free cholesterol in LDL challenged with ferrylmyoglobin during the experimental time (Fig. 5). It is also clear from Fig. 5 that the phenolic acids increased, up to $6 \mathrm{hr}$, the lag phase of $\mathrm{CO}$ disappearance, and, after the lag phases, clearly decreased the degradation rate of cholesteryl oleate. 


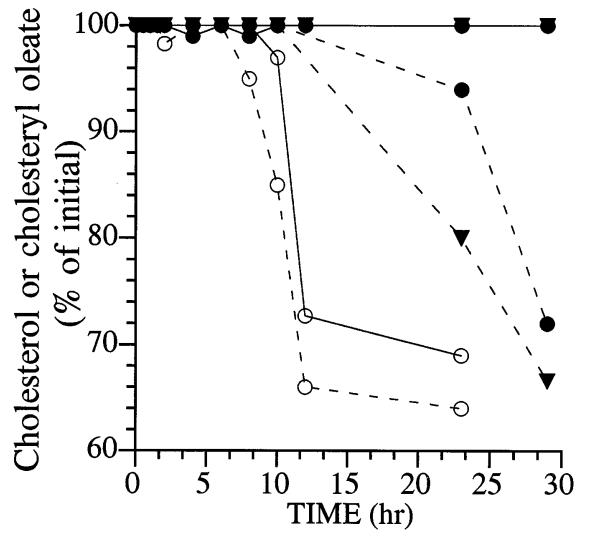

FIG. 5. Effects of caffeic and p-coumaric acids on free cholesterol and cholesteryl oleate contents in LDL oxidized as described in the legend of Fig. 1. Aliquots $(1 \mathrm{~mL})$ were removed for lipid extraction and HPLC analysis of free cholesterol (solid lines) and cholesteryl oleate (dashed lines). The concentrations of free cholesterol and cholesteryl oleate in starting LDL were 840 and $337 \mathrm{nmol} / \mathrm{mg}$ LDL protein, respectively. Symbols: $(\bigcirc)$ control (absence of phenolic acids), ( $)$ caffeic acid $(2 \mu \mathrm{M})$ and ( $\nabla)$ p-coumaric acid $(12 \mu \mathrm{M})$ added $3 \mathrm{~min}$ before starting oxidation.

\section{Synergistic Effect between Caffeic or p-Coumaric Acids with Ascorbate in Ferrylmyoglobin-Mediated LDL Oxidation}

In the isolated LDL fraction, not exposed to oxidation, $\mathrm{CL}-\mathrm{OOH}$ and 7-KC (a product of later peroxidation) were not detectable. In LDL incubated with ferrylmyoglobin, $\mathrm{CL}-\mathrm{OOH}$ content reached a maximum level at ca. $8 \mathrm{hr}$ (Fig. 6), but 7-KC reached a maximum level only after 22 hr incubation (Fig. 7). In Figs. 6 and 7 for the control assays (in absence of phenolic acids), lag phases for CL-OOH and $7-\mathrm{KC}$ were absent. However, in presence of caffeic $(2 \mu \mathrm{M})$ or p-coumaric $(12 \mu \mathrm{M})$ acids a lag phase of ca. 4 or $6 \mathrm{hr}$ was

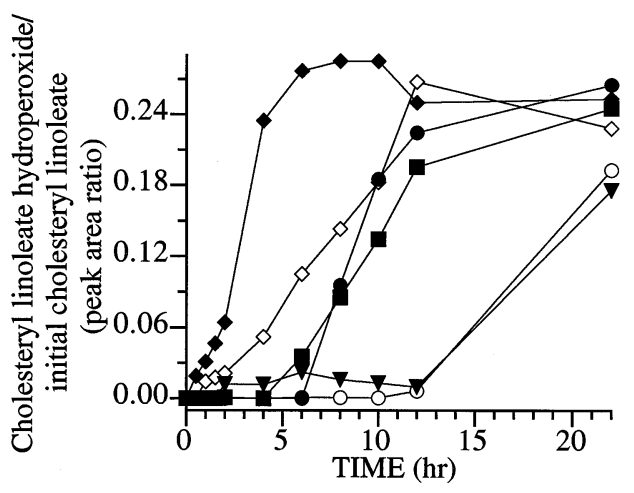

FIG. 6. Effects of caffeic or p-coumaric acids associated with ascorbate on the formation rate of cholesteryl linoleate hydroperoxide in LDL oxidized by ferrylmyoglobin. Symbols: $(\bullet)$ control (absence of antioxidants), $(\diamond)$ ascorbate $(4 \mu \mathrm{M}),(\square)$ caffeic acid $(2 \mu \mathrm{M}),(\bigcirc) 2 \mu \mathrm{M}$ caffeic acid plus $4 \mu \mathrm{M}$ ascorbate, (৩) p-coumaric acid $(12 \mu \mathrm{M})$ and $(\nabla) 12 \mu \mathrm{M}$ p-coumaric acid plus $4 \mu \mathrm{M}$ ascorbate added before starting oxidation. Cholesteryl linoleate hydroperoxide is expressed as indicated in the legend for Fig. 2.

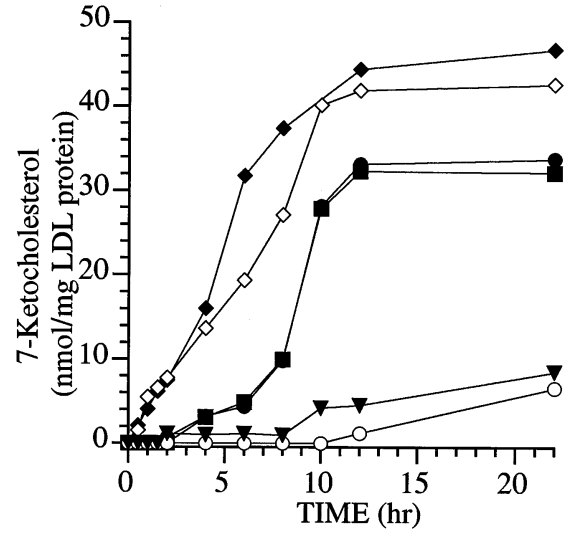

FIG. 7. Effects of caffeic or p-coumaric acids on 7-ketocholesterol formation in LDL exposed to ferrylmyoglobin in the absence and presence of ascorbate. LDL aliquots were extracted and analyzed by HPLC. Symbols: $(\diamond)$ control (absence of antioxidants), $(\diamond)$ ascorbate $(4 \mu \mathrm{M}),(\bigcirc)$ p-coumaric acid (12 $\mu \mathrm{M}),(\square)$ caffeic acid $(2 \mu \mathrm{M}),(\bigcirc) 2 \mu \mathrm{M}$ caffeic acid plus $4 \mu \mathrm{M}$ ascorbate and $(\nabla) 12 \mu \mathrm{M} p$-coumaric acid plus $4 \mu \mathrm{M}$ ascorbate added before oxidation.

clearly exhibited, in contrast to ascorbate that fails to induce an inhibition period of oxidation (Figs. 6 and 7). Moreover, the rates of formation of oxidized products decreased in presence of either phenolic acids or ascorbate. The protection afforded by ascorbate $(4 \mu \mathrm{M})$ on $7-\mathrm{KC}$ formation was considerably lower than that of caffeic or p-coumaric acids, at the concentrations used (Fig. 7). However, when caffeic or p-coumaric acids were added together with ascorbate, an inhibition period of oxidation of $\mathrm{ca} .12 \mathrm{hr}$ for the formation of CL-OOH was measured; this period was considerably longer than that expected from the sum of the periods observed in the presence of phenolic acids and ascorbate separately (Fig. 6). Similar effects were observed when the formation of 7-KC (Fig. 7) was measured. From this figure, it is clear that ascorbate potentiated the protection of either caffeic or p-coumaric acids against LDL oxidation, significantly increasing the inhibition periods of $7-\mathrm{KC}$ formation. Figures 6 and 7 also document that, after the lag phase, the formation rates of $7-\mathrm{KC}$ were depressed to a higher extent as compared to CL-OOH formation. On the other hand, the presence of caffeic and $p$-coumaric acids together induced inhibition periods not significantly different from the sum of periods induced by each phenolic acid separately (not shown).

Therefore, there exists a synergistic effect of the phenolic acids with ascorbate and a mere additive effect of the phenolic acids, in the absence of ascorbate, on LDL peroxidation challenged by ferrylmyoglobin.

\section{DISCUSSION}

The concentrations of lipid hydroperoxides in LDL in normal healthy subjects are rather low [17]. Although controversial, it has been reported that most plasma hydroperoxides are conveyed in LDL [21] rather than in high 
density lipoprotein fraction [22]. Several studies provide evidence for a robust positive correlation between the severity of atherosclerosis and plasmatic hydroperoxide concentration [23]. Moreover, the toxicity of oxidized LDL is apparently related to the amount of hydroperoxide carried by the oxidized LDL [24].

Ferrylmyoglobin initiates lipid peroxidation in several lipid systems, including LDL [25]. Since the most abundant cholesteryl ester in LDL is cholesteryl linoleate, measurement of its hydroperoxides is, in principle, a sensitive indicator of lipid peroxidation during oxidation of LDL by ferrylmyoglobin, and its suppression by dietary phenolic acids a clear indication of antioxidant activity.

The time-courses of cholesterol and cholesteryl ester depletion promoted by ferrylmyoglobin have been measured. Data indicate that ferrylmyoglobin is able to oxidize cholesteryl esters and free cholesterol in LDL, inducing a typical profile of LDL cholesteryl ester oxidation; the rate of oxidation is a function of unsaturation and follows the order: cholesteryl arachidonate $>$ cholesteryl linoleate $>$ cholesteryl oleate $>$ free cholesterol (Fig. 1). These results are consistent with those reported recently by Brown et al. [26] for $\mathrm{Cu}^{2+}$-oxidized LDL. Further, oxidation of LDL by ferrylmyoglobin or via azoperoxyl radicals (results not shown) yields identical product profiles representing cholesterol and cholesteryl esters loss or cholesteryl linoleate hydroperoxide formation, despite putative differences in the mechanisms of oxidation of these prooxidants.

In ferrylmyoglobin-challenged LDL, the cholesteryl linoleate hydroperoxide, not detectable in the original LDL fraction, increases in parallel with the loss of cholesteryl linoleate and, during the initial $2 \mathrm{hr}$ period, the decrease in $\mathrm{CL}$ is essentially related to the formation of CL-OOH (Fig. 2). Since CL-OOH is a major product of ferrylmyoglobincatalyzed LDL oxidation, it was used to evaluate the interplay between the phenolic acids and ascorbate on the protection of LDL from lipid peroxidation.

The health benefit of phenolic acids as antioxidants is a current interesting trend $[11,12]$. The plant metabolites caffeic and $p$-coumaric acids are chemically related (caffeic acid is a cathecol and p-coumaric is a monophenol) but exhibit very different antioxidant activities [13]. Caffeic and p-coumaric acids have a marked effect on the timecourses of cholesterol and cholesteryl esters degradation (Figs. 3A, 4 and 5) and the formation of cholesteryl linoleate hydroperoxide (Fig. 3B) in LDL oxidized by ferrylmyoglobin. These effects are expressed by increased lag times of linoleate hydroperoxide formation (Fig. 3B) and disappearance of cholesteryl esters (Fig. 3A) and also by a significant decrease in the rate of oxidation. Furthermore, the protective effect of these phenolic acids still persists after long periods of oxidative challenging (Fig. 4). It is particularly interesting that caffeic acid markedly inhibits ferrylmyoglobin-mediated LDL oxidation as compared with ascorbate at the same concentration. It was previously shown that ascorbate $[27,28]$, caffeic and $p$ coumaric acids [10] reduce ferrylmyoglobin back to met- myoglobin. Thus, the independent antioxidant activities of these compounds in suppression of ferrylmyoglobin-induced LDL lipid peroxidation are related to these reduction abilities. Caffeic acid is a better antioxidant than pcoumaric acid in ferrylmyoglobin-dependent LDL oxidation (Fig. 3A and B), a finding that is presumably related to the increased efficiency of caffeic over p-coumaric acid in reducing ferrylmyoglobin to metmyoglobin [10], a species harmless to LDL in absence of endogenous hydroperoxides. In agreement with this reduction ability, caffeic acid is a stronger scavenger of peroxyl radicals in the LDL system [12] as compared with $p$-coumaric acid.

The antioxidant activities of these compounds depend on the rate constants of the above-mentioned reactions and on the reactivity of phenoxyl radicals formed as products of the "antioxidant reaction". The p-coumaric acid is a less efficient radical scavenger than caffeic acid, a fact that may be ascribed to the increased radical stability of $o$-semiquinone radical of caffeic acid relative to the radical $p$ coumaric acid [29]. Recently, we reported that caffeic acid, like ascorbate, can regenerate $\alpha$-tocopherol from the $\alpha$ tocopheroxyl radical at the LDL surface but, in contrast, that $p$-coumaric acid potentiates $\alpha$-tocopherol consumption [13]. The p-coumaric behavior can be explained by the high reactivity of its phenoxyl radical (produced by an electron-transfer reaction between the phenolic acid and ferrylmyoglobin) with $\alpha$-tocopherol. Indeed, it was recently shown that simple phenoxyl radicals are highly reactive towards $\alpha$-tocopherol, oxidizing it [30]. Therefore, recycling of phenoxyl radicals from caffeic and $p$-coumaric acids by ascorbate, thereby yielding the very stable ascorbyl radical that terminates in the aqueous phase by disproportionation [31], would prevent eventual toxic effects of the phenolic compounds, increasing LDL protection against oxidation.

This synergistic interplay is well documented in Figs. 6 and 7 , on the basis of the following events: (a) the presence of ascorbate does not delay the onsets of $\mathrm{CL}-\mathrm{OOH}$ and 7-KC formation, but only slows down the rates; (b) when ascorbate is added together with caffeic or p-coumaric acids, the onsets are significantly delayed relative to the periods observed in the presence of phenolic acids alone. Ascorbate without effect, per se, increases the inhibition period of the phenolic acids from ca. $5 \mathrm{hr}$ to $12 \mathrm{hr}$. This event is presumably a consequence of phenolic acid regeneration promoted by ascorbate. Of course, this does not exclude the individual reactions of each of the three antioxidants in reducing ferrylmyoglobin to metmyoglobin, thus contributing to the overall antioxidant activity. The reaction between ascorbate and phenolic acids is kinetically (the compounds are located in the same compartment) and thermodynamically feasible (ascorbyl radical exhibits lower reduction potential than caffeic and p-coumaric radicals $[32,33])$.

Recent in vivo data have demonstrated that oxysterols, including 7-KC (a product of later lipid oxidation), accumulate in human foam cell macrophages from the athero- 
sclerotic plaque [34]. 7-KC is the major oxysterol present in ox-LDL-loaded cells, comprising approximately $60 \%$ of all oxysterols [34]. Gelissen et al. [35] reported that 7-KC may promote foam cell formation in vivo through inhibition of reverse sterol transport [35], a mechanism by which peripheral cells export excess cholesterol to an extracellular acceptor. Therefore, this compound is presumably critical to atherogenesis because it inhibits cholesterol removal from cells and is cytotoxic. In fresh LDL samples, 7-KC is not detectable and shows up along the time when LDL are exposed to ferrylmyoglobin. Therefore, ferrylmyoglobinmediated LDL oxidation generates advanced oxidation products of the type identified in human atherosclerotic plaque.

The 7-KC was particularly sensitive to the presence of metals, and its generation is apparently dependent on a redox cycle of metals [36]. Ferrylmyoglobin contains iron in the heme moiety, and at later oxidation steps can be released to the medium, thus potentiating $7-\mathrm{KC}$ formation. Therefore, since phenolic acids may bind transition metals [37], it is conceivable that ascorbate plus p-coumaric or caffeic and caffeic plus $p$-coumaric acids may suppress the generation of 7-KC more efficiently than that of $\mathrm{CL}-\mathrm{OOH}$.

In summary, LDL lipid peroxidation initiated by ferrylmyoglobin involves the degradation of cholesteryl esters as a function of unsaturation. The phenolic acids and ascorbate inhibit these reactions, the inhibition being strongly increased by the concerted activity of ascorbate with the phenols. Owing to the negative health impact of atherosclerosis in western societies, dietary phenolic acids may significantly improve public health as promising antiatherogenic agents. Further effort has to be made in terms of the bioavailabity and pharmacokinetics of these hydroxycinnamic plant metabolites. Since these phenolics occur ubiquitously in the plant kingdom, they are consumed in human diet in various amounts. Therefore, they may reach the plasma as a function of diet. In fact, a $\mathrm{Na}^{+}$-dependent saturable transport of cinnamic acid across the brush-border membrane of rat jejunum has recently been described [38]. This study provides in vitro evidence for a very effective mechanism contributing to the prevention of LDL lipid peroxidation that may have pathophysiological relevance in atherosclerosis: a synergistic antioxidant effect of caffeic and p-coumaric acids with ascorbate.

This work was supported by PRAXIS XXI (projects: 2/2.1/QUI/ $371 / 94$ and 2/2.1/BIO/1156/94). O. Vieira was supported by a grant (BD/5493/95) from PRAXIS XXI.

\section{References}

1. Berliner JA and Heinecke JW, The role of oxidized lipoproteins in atherosclerosis. Free Rad Biol Med 20: 707-727, 1996.

2. Esterbauer H, Gebicki J, Puhl H and Jurgens G, The role of lipid peroxidation and antioxidants in oxidative modification of LDL. Free Radic Biol Med 13: 341-390, 1992.

3. Stocker R, Bowry V and Frei B, Ubiquinol-10 protects human low density lipoprotein more efficiently against lipid peroxi- dation than does $\alpha$-tocopherol. Proc Natl Acad Sci USA 88: 1646-1650, 1991.

4. Noguchi N, Gotoh N and Niki E, Dynamics of the oxidation of low density lipoprotein induced by free radicals. Biochim Biophys Acta 1168: 348-357, 1993.

5. Steinbrecher U, Lougheed M, Kwan W and Dirks M, Recognition of oxidized low density lipoprotein by the scavenger receptor of macrophages results from derivatization of apolipoprotein B by products of fatty acid peroxidation. J Biol Chem 264: 15216-15223, 1989.

6. Smith L and Johnson B, Biological activities of oxysterols. Free Radic Biol Med 7: 285-332, 1989.

7. Rice-Evans C, Green E, Paganga G, Cooper C and Wrigglesworth J, Oxidised low density lipoproteins induce iron release from activated myoglobin. FEBS Lett 326: 177-182, 1993.

8. Giulivi C and Cadenas E, Ferrylmyoglobin: formation and chemical reactivity toward electron-donating compounds. Methods Enzymol 233: 189-202, 1994.

9. Laranjinha J, Vieira O, Almeida L and Madeira V, Inhibition of metmyoglobin $/ \mathrm{H}_{2} \mathrm{O}_{2}$ /-dependent low density lipoprotein lipid peroxidation by naturally occurring phenolic acids. Biochem Pharmacol 51: 395-402, 1996.

10. Laranjinha J, Almeida L and Madeira V, Reduction of ferrylmyoglobin by dietary phenolic acid derivatives of cinnamic acid. Free Radic Biol Med 19: 329-337, 1995.

11. Rive-Evans C, Miller NJ and Paganga G, Structure-antioxidant activity relationships of flavonoids and phenolic acids. Free Radic Biol Med 20: 933-956, 1996.

12. Laranjinha J, Almeida L and Madeira V, Reactivity of dietary phenolic acids with peroxyl radicals: antioxidant activity upon low density lipoprotein peroxidation. Biochem Pharmacol 48: 487-494, 1994.

13. Laranjinha J, Vieira $\mathrm{O}$, Madeira $\mathrm{V}$ and Almeida L, Two related phenolic antioxidants with opposite effects on vitamin E content in low density lipoproteins oxidized by ferrylmyoglobin. Arch Biochem Biophys 323: 373-381, 1995.

14. Vieira O, Laranjinha J, Madeira V and Almeida L, A rapid isolation of low density lipoproteins in a concentrated fraction free from water-soluble plasma antioxidants. J Lipid Res 37: 379-385, 1996.

15. Lowry O, Rosebrough A, Farr M and Randall R, Protein measurement with the Folin phenol reagent. J Biol Chem 193: 265-275, 1951.

16. Kritharides L, Jessup W, Gifford J and Dean T, A method for defining the stages of low-density lipoprotein oxidation by the separation of cholesterol- and cholesteryl ester-oxidation products using HPLC. Anal Biochem 213: 79-89, 1993.

17. Thomas J, Kalyanaraman B and Girotti A, Involvement of preexisting lipid hydroperoxides in $\mathrm{Cu}^{2+}$-stimulated oxidation of low-density lipoprotein. Arch Biochem Biophys 315: 244-254, 1994.

18. El-Saadani M, Esterbauer H, El-Sayed M, Goher M, Nassar A and Jurgens GJ, A spectrophotometric assay for lipid peroxides in serum lipoproteins using a commercially available reagent. J Lipid Res 30: 627-629, 1989.

19. Barclay LRC, Locke SJ, MacNeil JM, VanKessel J, Burton GW and Ingold KU, Autoxidation of micelles and model membranes. Quantitative kinetic measurements can be made by using either water-soluble or lipid-soluble initiators with water-soluble or lipid-soluble chain-breaking antioxidants. J Am Chem Soc 106: 2479-2481, 1984.

20. Frei B, England L and Ames B, Ascorbate is an outstanding antioxidant in human blood plasma. Proc Natl Acad Sci USA 86: 6377-6381, 1989.

21. Nourooz-Zadeh J, Tajaddini-Sarmadi J, Ling K and Wolff S, Low-density lipoprotein is the major carrier of lipid hydroperoxides in plasma. Biochem J 313: 781-786, 1996.

22. Bowry VW, Stanley KK and Stocker R, High density lipopro- 
tein is the major carrier of lipid hydroperoxides in human plasma from fasting donors. Differential distribution of ferulic acid to the major plasma constituents in relation to its potential as an antioxidant. Proc Natl Acad Sci USA 89: 10316-10320, 1992.

23. Stringer $M$, Gorog $P$, Freeman $A$ and Kakkar V, Lipid peroxides and atherosclerosis. Br Med J 298: 281-284, 1989.

24. Négre-Salvayre A, Lopez M, Levade T, Pieraggi M, Dousset N, Douste-Blazy L and Salvayre R, Ultra-treated lipoproteins as a model system for the study of the biological effects of lipid peroxides on cultured cells. II. Uptake and cytotoxicity of ultraviolet-treated LDL on lymphoid cell lines. Biochim Biophys Acta 1045: 224-232, 1990.

25. Dee G, Rice-Evans C, Obeyesekera S, Meraji S, Jacobs M and Bruckdorfer K, The modulation of ferrylmyoglobin formation and its oxidative effects on low density lipoproteins by nitric oxide. FEBS Lett 294: 38-42, 1991.

26. Brown A, Dean R and Jessup W, Free and esterified oxysterol: formation during copper-oxidation of low density lipoprotein and uptake by macrophages. J Lipid Res 37: 320-335, 1996.

27. Giulivi $\mathrm{C}$ and Cadenas $\mathrm{E}$, The reaction of ascorbic acid with different heme iron redox states of myoglobin: antioxidant and prooxidant aspects. FEBS Lett 332: 287-290, 1993.

28. Galaris D, Cadenas E and Hochstein P, Redox cycling of myoglobin and ascorbate: a potential protective mechanism against oxidative reperfusion injury in muscle. Arch Biochem Biophys 273: 497-504, 1989.

29. Bors W, Heller W, Michel C and Saran M, Flavonoids as antioxidants: determination of radical-scavenging efficiencies. Methods Enzymol 186: 343-355, 1990.

30. Foti $\mathrm{M}$, Ingold $\mathrm{K}$ and Lusztyk J, The surprising high reactivity of phenoxyl radicals. J Am Chem Soc 116: 9440-9447, 1994.
31. Roginsky VA and Stegmann HB, Ascorbyl radical as natural indicator of oxidative stress: quantitative regularities. Free Radic Biol Med 17: 93-103, 1994.

32. Jovanovic S, Steeken S, Tosic M, Marjanovic B and Simic M, Flavonoids as antioxidants. J Am Chem Soc 116: 4846-4851, 1994.

33. Steenken S and Neta P, One-electron redox potentials of phenols. Hydroxy- and aminophenols and related compounds of biological interest. J Phys Chem 86: 3661-3667, 1982.

34. Hultén L, Lindmark H, Diczfalusy U, Bjorkhem I, Ottosson M and Liu YJ, Oxysterols present in atherosclerotic tissue decrease the expression of lipoprotein lipase messenger RNA in human monocyte-derived macrophages. J Clin Invest 97: 461-468, 1996.

35. Gelissen I, Brown A, Mander E, Kritharides L, Dean R and Jessup W, Sterol efflux is impaired from macrophage foam cells selectively enriched with 7-ketocholesterol. J Biol Chem 271: 17852-17860, 1996

36. Kritharides L, Jessup and Dean RT, EDTA differentially and incompletely inhibits components of prolonged cell-mediated oxidation of low-density lipoprotein. Free Radic Res 22: 399-417, 1995.

37. Iwahashi $H$, Ishii $T$, Sugata $R$ and Kido $R$, The effects of caffeic acid and its related catechols on hydroxyl radical formation by 3-hydroxyanthranilic acid, ferric chloride, and hydrogen peroxide. Arch Biochem Biophys 276: 242-247, 1990.

38. Wolffram S, Weber T, Grenacher B and Scharrer E, $\mathrm{Na}^{+}$, dependent mucosal uptake of cinnamic acid across the jejunal brush border in rats. J Nutrition 125: 1300-1308, 1995. 